\title{
Twenty two year survival after incomplete resection of advanced adenoid cystic bronchogenic carcinoma
}

\author{
N. Schoenfeld*, W. Rahn**, R. Loddenkemper*
}

\begin{abstract}
Twenty two year survival after incomplete resection of advanced adenoid cystic brochogenic carcinoma. N. Schoenfeld, W. Rahn, R. Loddenkemper. @ERS Journals 1996. ABSTRACT: In 1972, a 42 year old patient underwent thoracotomy for adenoid cystic carcinoma of the left main-stem bronchus. Pneumonectomy was performed, but the resection was not complete as the tumour had invaded the aortic and tracheal wall. The patient did not receive postoperative radiotherapy.

In 1993, the patient was readmitted with severe respiratory insufficiency due to complete endobronchial tumour obstruction of the intermediate bronchus and extensive tumour growth in the mediastinum. Endobronchial laser treatment followed by stent implantation led to immediate symptomatic relief. The tumour then responded well to combined endobronchial and percutaneous radiotherapy. The patient died 7 months after readmission from ileus due to pancreatitis.

We conclude that patients with advanced adenoid cystic carcinoma may profit from palliative surgery with respect not only to symptoms but also to duration of life.

Eur Respir J., 1996, 9, 1560-1561.
\end{abstract}

\author{
*2nd Department of Pulmonary Medicine \\ and **Institute of Pathology, Lungenklinik \\ Heckeshorn, Berlin, Germany. \\ Correspondence: N. Schoenfeld \\ Pneumologische Abt. II \\ Lungenklinik Heckeshorn \\ Zum Hecheshorn 33 \\ 14109 Berlin \\ Germany \\ Keywords: Adenoid cystic carcinoma \\ bronchogenic carcinoma \\ laser therapy \\ radiotherapy \\ surgery
}

Received: April 191995

Accepted after revision November 211995
Adenoid cystic carcinoma of the bronchial system is a rare malignancy usually located in the central airways, i.e. the trachea or main-stem bronchi [1]. It appears to be unrelated to smoking, with an equal distribution in males and females. The tumour most often infiltrates the adjacent structures in continuity. Metastases to regional lymph nodes or distant sites are less common. Long-term survival has been reported for patients when the tumour has been resected $[2,3]$, but local recurrences of tracheal tumours have been recorded even after 25 and 30 yrs [4].

\section{Case report}

A 42 year old male nonsmoker was admitted in December 1971 with almost complete atelectasis of the left lung. Bronchoscopy revealed an obstructing tumour in the proximal left main-stem bronchus. Thoracotomy showed that the tumour had grossly invaded peribronchial structures, including the aortic wall and the tracheal wall. Numerous enlarged lymph nodes were found. Pneumonectomy was performed, but residual tumour remained in the aortic wall and the bronchial surgical margins.

Histologically, adenoid cystic carcinoma was diagnosed. The tumour showed trabecular and tubular structures filled with alcian blue- and periodic-acid-Schiff (PAS)-positive mucin. The surgical margins were positive, but none of the enlarged lymph nodes were involved by the tumour (tumour, node, metastasis classification (TNM) stage pT4NOM0 [5]). As an additional finding, epi-thelioid cell granulomatas with necrosis were detected within the resected lung, suggesting pulmonary tuberculosis. Antituberculosis treatment with isoniazid and ethambutol was given for 18 months; sputum cultures remained negative. The patient did not receive postoperative radiotherapy.

In May 1993, the patient was readmitted with a 2 week history of severe respiratory insufficiency at rest. Bronchoscopy revealed complete tumour obstruction of the whole length of the intermediate bronchus, and extrabronchial compression of the right main-stem bronchus. Histologically, the tumour was again identified as adenoid cystic carcinoma of tubular subtype (fig. 1). Computed tomography showed extensive tumour growth in the mediastinum. The intermediate bronchus was reopened by laser resection followed by the insertion of a tandem

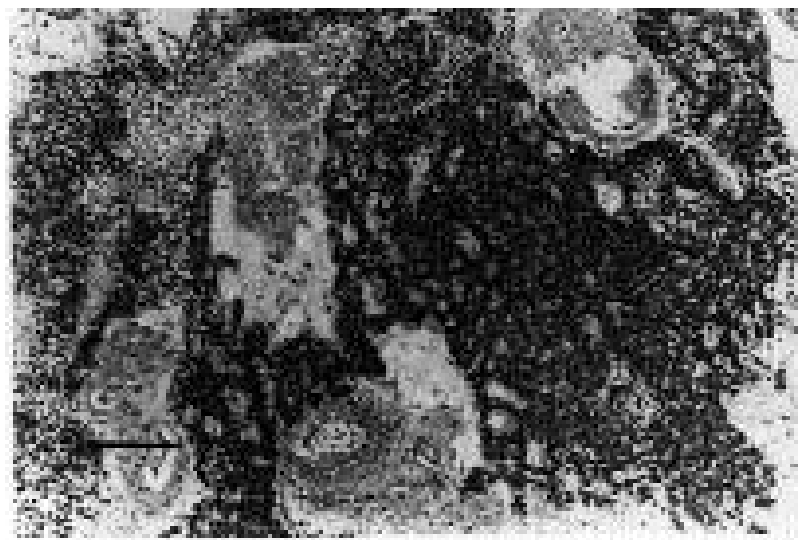

Fig. 1. - Tubular subtype of adenoid cystic carcinoma. The biopsy was taken during bronchoscopy when the patient was readmitted 22 yrs after pneumonectomy. (Haemtoxylin and eosin stain; internal scale bar $=0.3 \mathrm{~mm}$ ). 
Gianturco expandable metallic stent into the right mainstem bronchus, which led to immediate relief of symptoms. Endobronchial radiation in the intermediate and mainstem bronchus was given in three doses with 15 Gy every 2 weeks, and 30 Gy were given percutaneously within 7 weeks in weekly fractions, achieving partial remission of the tumour in the mediastinum. However, metastatic tumour growth developed during follow-up, with a single nodule in the right lower lobe.

When endobronchial treatment had been completed the patient was able to live at home with a good quality of life. He died unexpectedly in December 1993 from sudden ileus due to pancreatitis, without evidence of underlying disease. Unfortunately, no permission was given for an autopsy.

\section{Discussion}

Adenoid cystic carcinoma of the bronchial system can be divided into three histological subtypes, as in the salivary glands: a tubular-trabecular; an intermediate cribriform-microcystic; and a poorly differentiated type [1]. The latter has the largest solid component. Whereas MoRAN et al. [4] did not find a correlation between histological features and clinical behaviour, NOMORI et al. [3], described prognostic differences of the different tumour types, indicating a poorer prognosis for the solid subtype due to metastatic spread as has been described for salivary gland tumours. The data of MoRAn et al. [4] suggested that the disease stage at the time of diagnosis may play a role in predicting the outcome.

A few cases have been described with tumour recurrence decades after complete resection $[2,6]$. In the series of NomORi et al. [3], survival times after surgery ranged 41-87 months, including patients with and without tumour at surgical resection margins. In our case, the patient had advanced disease with a T4 tumour, but he survived 22 yrs after incomplete resection and without postoperative radiotherapy. Although our patient had adenoid cystic carcinoma of the tubular subtype, he developed intrapulmonary metastases during the late course of the disease.
Laser resection is a well-established therapeutic tool in the palliation of endobronchial low-grade malignancies [7]. In localized adenoid cystic tumours a symptomfree survival of 4-6 months after laser therapy has been reported [7]. Combined treatment modalities, including laser resection, stent implantation and endobronchial radiotherapy, were applied in this patient with good results until he died 7 months later of unrelated conditions. As described in other cases, external beam radiation was also effective in our patient [1].

In conclusion, we assume that even in unresectable adenoid cystic carcinoma palliative surgery can be part of a reasonable therapeutic concept, which may lead not only to symptomatic relief but also to prolongation of the patient's life, in contrast to other more common types of bronchogenic carcinoma. Endobronchial recurrences can be treated efficiently with interventional bronchoscopic measures.

\section{References}

1. Dail DH. Uncommon tumors. In: Dail DH, Hammar SP, eds. Pulmonary Pathology. 3rd edn. Berlin, Heidelberg, New York, Springer, 1994: pp. 1279-1462.

2. Houston HE, Payne WS, Harrison EG Jr, Olsen AM. Primary cancers of the trachea. Arch Surg 1969; 99: 132-140.

3. Nomori H, Kaseda S, Kobayashi K, Ishihara T, Yanai $\mathrm{N}$, Torikata C. Adenoid cystic carcinoma of the trachea and bronchus in a general hospital. J Thorac Cardiovasc Surg 1988; 96: 271-277.

4. Moran CA, Suster S, Koss MN. Primary adenoid cystic carcinoma of the lung. Cancer 1994; 73: 1390-1397.

5. International Union Against Cancer. TNM classification of malignant tumors. 4th edn. Geneva, International Union Against Cancer, 1987: 69-73.

6. Wilkins EW, Darling RC, Soutter L, Sniffen RC. A continuing clinical survey of adenomas of the trachea and bronchus in a general hospital. J Thorac Cardiovasc Surg 1963; 46: 279-291.

7. Diaz-Jiménez JP, Canela-Cardona M, Maestre-Alcacer J. $\mathrm{Nd}$ :YAG laser photoresection of low-grade malignant tumors of the tracheobronchial tree. Chest 1990; 97: 20-22. 